\title{
Methotrexate in patients with moderate systemic lupus erythematosus (exclusion of renal and central nervous system disease)
}

\author{
S Gansauge, A Breitbart, N Rinaldi, M Schwarz-Eywill
}

\begin{abstract}
Objectives-Methotrexate (MTX) has been used in several autoimmune diseases. Apart from its use in rheumatoid arthritis, MTX has been assessed in small studies in patients with vasculitis, uveitis, and inflammatory bowel disease. The aim of this study was to evaluate the efficacy of MTX in a particular group of patients with systemic lupus erythematosus (SLE). Patients-In an open prospective study 22 patients fulfilling the ACR criteria for SLE were included. Patients had one or more of the following manifestations: active non-destructive polyarthritis, dermatitis, vasculitis of the skin, pleuritis. All patients had been treated with corticosteroids for at least six months without achieving remission. Sixteen patients were taking antimalarial drugs in addition to corticosteroids, which were stopped at the beginning of the trial. Patients with renal and central nervous involvement were excluded from the study. All patients received MTX orally at a dose of 15 mg/week over six months. Corticosteroids were continued. As additional medication only indomethacin up to $100 \mathrm{mg} /$ day was permitted if used before the start of the study. The outcome was evaluated using the SLE disease activity index (SLEDAI). Results-Disease activity was evaluated after six months of MTX treatment. All patients completed the study period. The SLEDAI decreased significantly from mean (SD) $12.2 \quad(3.99)$ to $4 \quad(3.75)$ $(p=0.001)$. The prednisolone dose was reduced from a mean (SD) of $17.4(12.8)$ at the beginning to $8.8(5.36) \mathrm{mg} / \mathrm{day}$ at the end point of the study $(p=0.01)$. MTX was well tolerated. Four patients complained of general malaise. Two patients had transient increases in liver enzymes. In no case did MTX have to be stopped.

Conclusions-In an open prospective study methotrexate was used in SLE patients with particular clinical characteristics. MTX was shown to be effective in reducing disease activity and sparing the dose of corticosteroids. Further controlled studies are necessary.
\end{abstract}

(Ann Rheum Dis 1997;56:382-385)

Dr S Gansauge, St Vincen und Elisabeth Hospital, Abteilung Rheumatologie, An der Goldgrube 11, 55131 Mainz, Germany.

Accepted for publication 10 March 1997

Universität

A Breitbart

M Schwarz-Eywil

St Vincenz und Elisabeth Hospital, Mainz, Germany

$S$ Gansauge

Methotrexate (MTX) has been successfully used in several rheumatological diseases, such as rheumatoid arthritis, psoriatic arthritis,
Reiter's syndrome, and more recently vasculitis. ${ }^{1}$ The first data concerning successful use of MTX in systemic lupus erythematosus (SLE) were published as early as 1965 by Miescher $e t a l^{2}$ but it is only recently that interest has grown. Recent publications have shown the usefulness of MTX in SLE pointing out that MTX might be an alternative to conventional treatment with high dose corticosteroids, azathioprine or alkylating substances such as cyclophosphamide..$^{3-10}$ In contrast with cyclophosphamide and azathioprine oncogenicity has not been known for MTX. Without doubt treatment with corticosteroids is very effective for SLE, but in longstanding disease complications such as infections and osteoporosis are worrying.

Because of the paucity of published data it is uncertain which patients actually profit from MTX therapy. For the many patients with mild disease who are well managed with symptomatic treatment and antimalarial drugs, there is certainly no need for intensification of treatment. In lupus nephritis ${ }^{11-13}$ several well conducted studies have shown the benefit of cyclophosphamide and corticosteroids and this is the established regimen nowadays. However there remains a group of patients with moderate non-life threatening disease, which nevertheless has a high morbidity. The purpose of this study was to investigate if these patients might profit from low dose methotrexate treatment.

\section{Methods}

PATIENTS

All patients had an established diagnosis of SLE as defined by the American College of Rheumatology 1982 criteria. $^{14}$ Inclusion criteria were as follows: (1) refractory cutaneous rashes, (2) active vasculitis of the skin, (3) active pleurisy, (4) active arthritis. Vasculitis of the skin was defined as ulceration, gangrene, tender finger nodules, periungual infarction, splinter haemorrhages or histological proof of vasculitis. Patients with evidence for active lupus nephritis or central nervous system involvement were excluded. In addition patients were excluded if they had impaired renal function (creatinine clearance $<80$ $\mathrm{ml} / \mathrm{min}$ ) or abnormal liver enzyme activity. Female patients of child bearing age who were not practising adequate contraceptive measures were also excluded. Alcohol was proscribed.

Patients were eligible if they had been treated with more than $7.5 \mathrm{mg}$ prednisolone 


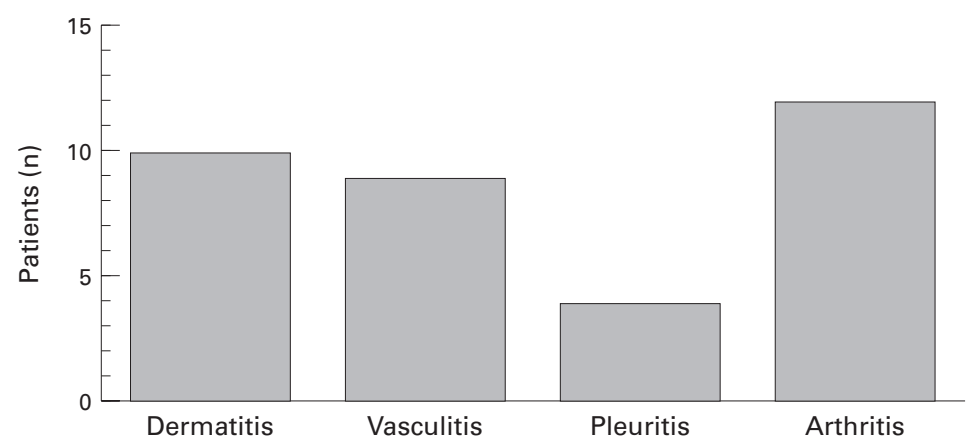

Figure 1 Disease manifestations present at the time of study entry.

for at least six months without achieving remission. Corticosteroid treatment was continued, previous therapy with antimalarial drugs was stopped at the beginning of the trial. For all patients who had been taking indomethacin before the start of the study, indomethacin in a dose of $100 \mathrm{mg} /$ day was permitted as well. Other non-steroidal antiinflammatory drugs were not used.

STUDY DESIGN

MTX was given in a standard dose of $15 \mathrm{mg}$ orally per week, folic acid was not given on a routine basis. For six months patients were reviewed every four weeks and had clinical, laboratory, and immunological assessement.

Disease activity was recorded according to the SLE disease activity index (SLEDAI) ${ }^{1516}$ initially and after six months. Standard laboratory tests, erythrocyte sedimentation rate (ESR), complement components C3c, C4 (determined by nephelometry), and anti-DNA antibody values (as determined by Farr assay) were assessed every four weeks.

STATISTICAL ANALYSIS

Student's two tailed $t$ test for paired samples was used for comparisons. The Wilcoxon matched pairs signed rank test was substituted if the distribution of the differences between paired data deviated substantially from normal or in case of non-parametric values (SLEDAI). For both statistical methods $\mathrm{p}$ values less than 0.05 were considered significant.

\section{Results}

Nineteen patients were female, three were male. The mean age was 41 years (range 24 68). Disease duration ranged from 1 to 13 years before MTX therapy. The mean (SD) corticosteroid dose at entry in the study was 17.4 (12.8) $\mathrm{mg}$ prednisolone/day. In 16 patients previous treatment with antimalarial drugs had been ineffective. Figure 1 shows the clinical manifestations at the time of study entry. MTX was given as a means to control arthritis in 12 patients, dermatitis in 10 patients, pleurisy in four patients, and vasculitis of the skin in nine patients. Often more than one symptom was present at the time MTX was started. The mean number of symptoms at the start of treatment was 1.45 with a range of 1-3. Mean (SD) SLEDAI was 12.2 (3.99).
In all cases dsDNA antibodies were positive. Complement components $\mathrm{C} 3 \mathrm{c}, \mathrm{C} 4$ were decreased in 12 patients and 10 had normal $\mathrm{C} 3 \mathrm{c}, \mathrm{C} 4$ values.

All patients finished the study period. Figures 2 and 3 show the changes in clinical disease activity (SLEDAI), prednisolone dose, and laboratory data. SLEDAI decreased significantly from 12.2 (SD 3.99) to 4 (3.75) $(p=0.001)$. The mean (SD) corticosteroid dose was significantly reduced from $17.4(12.8)$ to 8.8 (3.99) $(\mathrm{p}=0.01)$. In detail disappearance of symptoms was noted in 10 of 12 patients with arthritis, 8 of 10 patients with dermatitis, 3 of 4 patients with pleuritis, and 6 of 9 patients with vasculitis of the skin. Disease activity was completely suppressed in 16 patients (SLEDAI score 2) and was considerable reduced in four patients (SLEDAI score 3-6). These patients were defined as responders. Ten of 12 patients with arthritis had a SLEDAI score of 2 at the end of the study period. SLEDAI score did not change in two patients, these patients were classified as non-responders, both having refractory vasculitic ulcers.

Initial clinical improvement and a trend toward lower doses of corticosteroids was seen after four to eight weeks, but that trend was only statistically significant at six months.

The mean values of complement components $\mathrm{C} 3 \mathrm{c}, \mathrm{C} 4$ did not change significantly in patients with normal values before MTX treatment (normal range $0.55-1.2$ ). In patients with decreased $\mathrm{C} 3 \mathrm{c} / \mathrm{C} 4$ values changes were more evident but differences were still not significant.

Mean (SD) ESR decreased significantly from $46(21)$ to 32 (13) in the first hour ( $p=$ 0.02 ). There was a reduction of dsDNA antibody concentrations, but differences were not significant.
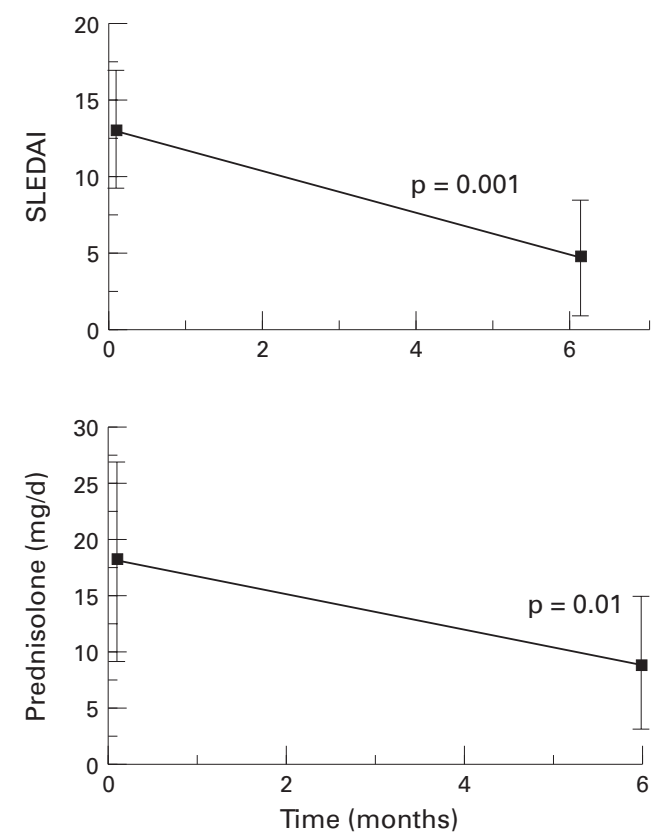

Figure 2 Reduction of disease activity and corticosteroid dose after six months of MTX treatment. 

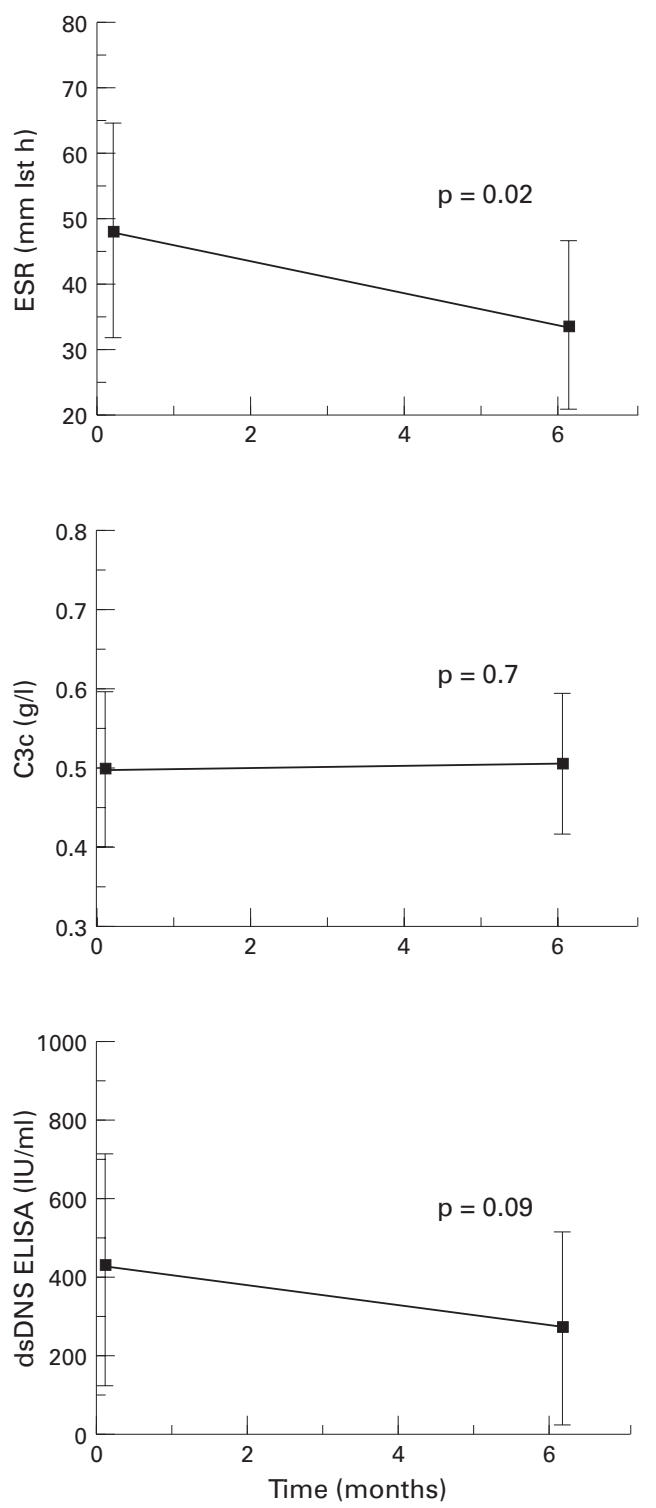

Figure 3 Laboratory changes after six months of MTX treatment.

ADVERSE REACTIONS

Four patients suffered from general malaise, mainly soon after starting MTX treatment, in two patients a small increase in liver enzyme activities was observed (less than twice the normal values). Malaise and increase in liver enzyme activities did not warrant discontinuation of MTX treatment. As malaise resolved after the second or third dose of MTX, patients were not given folic acid supplementation.

\section{Discussion}

In 1965 Miescher and Riethmüller ${ }^{2}$ used MTX in 10 SLE patients with joint pain, skin eruption, and vascular purpura at a dose of 50 $\mathrm{mg} /$ week intravenously. These investigators noted a rapid response and manifestations of SLE diminished within one or two weeks. Swanson and Schwartz ${ }^{17}$ later reported a response to MTX in four SLE patients. In contrast Dubois ${ }^{18}$ noted no benefit or toxicity of MTX in seven patients with SLE, of whom six had lupus nephropathy. More recently
Rothenberg and coworkers ${ }^{3}$ examined the response to MTX in 10 patients with corticosteroid resistant SLE. Of 10 patients treated with MTX at a dose of $7.5 \mathrm{mg} /$ weekly, seven showed improvement. Features that improved included myositis, rash, arthritis, pleurisy, and proteinuria. Wilke et $a l^{4}$ reported a retrospective study of 17 unselected cases with SLE who were treated with oral MTX for arthritis, rash, serositis, fatigue, and renal disease. MTX treatment resulted in symptomatic improvement in $57 \%$ of patients and the corticosteroid dose was decreased by half. More recently Walz Le Blanc et $a l^{10}$ reported the beneficial effect of MTX in five patients, three with renal disease and two with arthritis. In an open ended trial Wilson and Abeles ${ }^{5}$ treated 12 patients with SLE. Arthritis was the major persistent problem in seven patients, one patient had recurrent pleuropericarditis, two patients had refractory cutaneous lupus rashes, and two had vasculitis. Three patients stopped MTX treatment because of side effects. In eight of nine remaining patients the glucocorticoid dose was reduced by an average of $42 \%$. Arfi $e t a l^{6}$ reported an open prospective study in a group of 16 patients who presented SLE with cutaneous and articular symptoms, and who required treatment with a minimum dose of $15 \mathrm{mg}$ prednisone per day. MTX was given in a dose of $7.5 \mathrm{mg}$ intramuscularly per week. Improvement was observed in 13 of 16 patients and permitted the reduction of the amount of prednisone required.

We have evaluated the response to MTX treatment in 22 patients with non-renal SLE suffering from active arthritis, dermatitis, vasculitis of the skin or pleuritis. After a study period of six months, we found a significant decrease in disease activity as measured by SLEDAI and a significant reduction of daily corticosteroid dose. MTX was well tolerated and major side effects leading to discontinuation were not seen during the study period. Two patients did not respond to MTX treatment both having refractory vasculitic ulcers. The best response was seen in patients with active arthritis with complete suppression of disease activity in 10 of 12 cases. Of the laboratory parameters only the decrease in ESR was significant. Despite a slight decrease in dsDNA antibody concentrations changes were not significant. MTX did not influence the complement values significantly. This might be because the mean complement values were already within normal ranges before the start of MTX treatment as most patients showed only moderate disease activity compared with those with, for example, severe lupus nephritis or central nervous system involvement.

In every treatment trial of SLE, one has to consider that the variable course of the disease makes the interpretation of any drug efficacy very difficult. The nature of lupus activity in our patients before MTX treatment makes the possibility of spontaneous improvement less likely, reduction in the prednisolone dose also suggests that the MTX was effective. 
The question whether SLE patients with renal or central nervous involvement may benefit from MTX treatment needs to be investigated very cautiously. As treatment failure in this high risk patient group may result in life threatening situations, we would recommend the well established treatment with cyclophosphamide.

We feel that there is a role for MTX in moderate SLE. The failure of treatment with antimalarial drugs often results in long term treatment with higher doses of corticosteroids. The data support the hypothesis that MTX can be used as significant corticosteroid sparing agent reducing further morbidity related to therapy. Even though alkylating substances are known to be effective and have a potential as corticosteroid sparing agents, there is concern about proved or possible risks of carcinogenicity or irreversible sterility. Our experience and that of others suggests that MTX is well tolerated and effective in SLE. To date no evidence of oncogenicity has been reported, however, the lack of oncogenicity of MTX is somewhat controversial in rheumatoid arthritis patients and is certainly not able to be confirmed in patients with systemic lupus at the present time as there is no long term experience with a large group of lupus patients taking MTX. Nevertheless MTX may be preferable to azathioprine on the grounds of decreased oncogenicity in young patients, keeping in mind the potential for serious toxicity in the event of compromise of renal function. As six months is a comparatively short period in the course of the disease further studies with long term follow up are necessary. To improve and extend therapeutic strategies further investigation of the use of MTX in SLE is warranted, emphasising a stage adapted therapeutic regimen. Further randomised double blind controlled studies are needed to compare MTX with other immunosuppressive agents in this subgroup of patients with moderate non-renal SLE.

We thank Dr David Maclachlan (Medizinische Klinik un Poliklinik V, Universität Heidelberg) for helpful comments on the manuscript and Dario R C Brocai (Stiftung Orthopädische Universitätsklinik Heidelberg) for statistical analysis of the data.

1 Sneller MC, Hoffman GS, Talar-Williams C, Kerr GS, Hallahan CW, Fauci AS. An analysis of forty-two Wegener's granulomatosis patients treated with methotrexate and prednisone. Arthritis Rheum 1995;38:608-13.

2 Miescher PA, Riethmüller D. Diagnosis and treatment of systemic lupus erythematosus. Semin Hematol 1965;2:1-

3 Rothenberg RJ, Graziano FM, Grandone JT, Goldberg JW, Bjarnason DF, Finesilver AG. The use of methotrexate in steroid-resistant systemic lupus erythematosus. Arthritis Rheum 1988;31:612-5.

4 Wilke WS, Krall PL, Scheetz RJ, Babiak T, Danao T, Mazanec DJ, et al. Methotrexate for systemic lupus erythematosus: A retrospective analysis of 17 unselected cases. Clin Exp Rheumatol 1991;9:581-7.

5 Wilson K, Abeles M. A 2 year, open ended trial of methotrexate in systemic lupus erythematosus. J Rheumatol 1994:21:1674-7.

6 Arfi S, Numeric P, Grollier L, Panelatti G, Jean-Baptiste G. Tratement du lupus erythemateux corticodependant par le methotrexate a faible doses. Rev Med Interne 1995;16: 885-90.

7 Guil-Garcia M, Garcia-Potales R, Fernandez-Nebro A, Belmonte-Lopez A, Camps-Garcia M T, Ramon-Garrido
E. The efficacy of methotrexate in systemic lupus E. The efficacy of methotrexate in systemic
erythematosus. Med Clin (Barc) 1993;101:361-4.

8 Bottomley W, Goodfield M. Methotrexate for severe cutaneous lupus erythematosus. Br J Dermatol 1993;129 (suppl 42):36.

9 Fox DA, McCune WJ. Immunosuppressive therapy of systemic lupus erythematosus. Rheum Dis Clin North Am 1994;20:265-99.

10 Walz LeBlanc BAE, Dagenais P, Urowitz MP, Gladman D D. Methotrexate in systemic lupus erythematosus. J Rheumatol 1994;21:836-8.

11 Mills JA. Systemic lupus erythematosus. N Engl J Med 1994;330:1871-9.

12 Austin HA, Klippel JH, Balow JE, Le Riche NGH, Steinberg AD, Plotz PH, et al. Therapy of lupus nephritis: controlled trial of prednisone and cytotoxic drugs. N Engl J Med 1986;314:614-9.

13 Boumpas DT, Austin HA, Vaughan EM, Klippel JH, Steinberg AD, Balow JE. Severe lupus nephritis: controlled trial on pulse methylprednisolone versus two different region pulse methylprednisolone versus two different regi-
mens of pulse cyclophosphamide. Lancet 1992; mens of

14 Tan EM, Cohen AS, Fries JF, Masi AT, McShane DJ, Rothfield NF, et al. The 1982 revised criteria for the classification of systemic lupus erythematosus. Arthritis Rheum 1982; 25:1271-7.

15 Liang $\mathrm{MH}$, Socher SA, Larson MG, Schur PH. Reliability and validitiy of six systems for the clinical assessment of disease activity in systemic lupus erythematosus. Arthritis Rheum 1989;32:1107-18.

16 Bombardier C, Gladman DD, Urowitz MB, Caron D, Chang $\mathrm{CH}$, and the Committee on Prognosis Studies in SLE. Derivation of the SLEDAI - a disease activity index for lupus patients. Arthritis Rheum 1992;35:630-40.

17 Swanson MA, Schwartz RS. Immunosuppressive therapy: the relation between clinical response and immunological competence. N Engl J Med 1967;277:163-70.

18 Dubois EL. Lupus erythematosus: a review of the current status of discoid and systemic lupus erythematosus and their variants. Los Angeles: University of California Press, 1974. 